Voix et Images

volxetimages

\title{
De l'autre côté du miroir... ou de la fenêtre
}

\section{Yvette Francoli}

Volume 10, numéro 3, printemps 1985

André Major

URI : https://id.erudit.org/iderudit/200526ar

DOI : https://doi.org/10.7202/200526ar

Aller au sommaire du numéro

\section{Éditeur(s)}

Université du Québec à Montréal

\section{ISSN}

0318-9201 (imprimé)

1705-933X (numérique)

Découvrir la revue

Citer ce compte rendu

Francoli, Y. (1985). Compte rendu de [De l'autre côté du miroir... ou de la fenêtre]. Voix et Images, 10(3), 201-203. https://doi.org/10.7202/200526ar d'utilisation que vous pouvez consulter en ligne.

https://apropos.erudit.org/fr/usagers/politique-dutilisation/ 


\title{
Essai \\ De l'autre côté du miroir... ou de la fenêtre
}

\author{
par Yvette Francoli, Université de Sherbrooke
}

Dans ce brillant essai, Maurice Émond ${ }^{1}$ tente de percer une fenêtre dans l'univers imaginaire d'Anne Hébert, à partir de ses premières ouvres romanesques, les Chambres de bois, Kamouraska, et les Enfants du Sabbat. Le lecteur entreprend avec lui cette longue traversée de l'autre côté du miroir - ou de la fenêtre - au hasard des dédales labyrinthiques de cette «autre terre vivante», véritable sous-sol de la conscience et de sa faune hagarde. Là, règne et sévit la «Mère terrible», cette «Fleur vénéneuse absolue de la nuit», qui exerce sa fascination pour mieux traquer sa victime et se jouer d'elle.

Cette analyse thématique qui s'inspire des méthodes critiques de Bachelard, Gilbert Durand, Jean-Pierre Richard, Georges Poulet, est menée et structurée avec une cohérence rigoureuse. Écrite dans une langue claire et directe, elle doit autant au grand souci d'objectivité de son auteur qu'à sa pénétrante intuition. La femme à la fenêtre s'articule autour de trois grands axes: une symbolique du noir et du blanc, une dialectique de l'eau et du feu et une poétique du regard qui, tout en dessinant les contours de l'imaginaire d'Anne Hébert, nous en signale également les constantes et les lignes de force. Maurice Émond a relevé systématiquement et avec beaucoup de minutie, tout un réseau d'images, de thèmes et de motifs portant sur les couleurs, les odeurs, la lumière, les ténèbres, les liens, les regards, les fenêtres et les miroirs, dont leur récurrence à travers l'oeuvre hébertienne prouve - pour reprendre les termes de Jakobson - que «seule l'existence d'éléments invariants permet de reconnaître les variations».

Toutes ces images s'inscrivent dans un double régime «diurne» et «nocturne» où se jouent les complicités du bien et du mal. Rêverie d'un monde déchiré entre les puissances du soleil et de la lune, de l'ombre et de la lumière, où dominent le noir et le blanc, couleurs manichéennes par excellence (auxquelles on pourrait ajouter les ors et les rouges, couleurs infernales de l'appartement de Hélö̈se). Noir et blanc comme un échiquier où se livre le dur combat de la vie et de la mort, des forces du bien et du mal, du rêve et de la réalité. Car, ainsi que le démontre Maurice Émond, les héros d'Anne Hébert sont la proie d'une culpabilité empreinte de terreur religieuse qui leur fait perdre le contrôle de la réalité pour vivre le monde halluciné de la faute. On verra, par exemple, le couple adultère et criminel, Georges et Élisabeth, de Kamouraska, refuser toute vision familière et objective des choses pour vivre les terreurs nocturnes d'une conscience qui rêve et qui souffre. C'est alors, comme cette étude le met en évidence, que les ténèbres s'animent de figures bestiales et que les héros, dans l'enfer de leur nuit, en viennent à s'identifier eux-mêmes à quelque oiseau nocturne ou bête féroce. Élisabeth, qui croit voir à tout moment surgir de l'ombre une 
inquiétante "bête sauvage», découvre soudain chez Georges de «fortes et longues dents», trait physique jusque-là passé inaperçu. Ce thème de la bête féroce - parmi tant d'autres qu'il nous est impossible de tous évoquer ici se répète avec Soeur Julie aux «dents blanches et fortes qui se retroussent» et aux yeux dont «la pupille est horizontalement fendue comme celle des loups». Et, comme une image en appelle une autre, soeur Julie «la possédée» se met à rôder la nuit dans les caves du couvent - sombres enfers - à la recherche de viande crue et de sang. C'est avec beaucoup de justesse que Maurice Émond fait remarquer que ces images récurrentes qui servent à alimenter les récits d'Anne Hébert et orienter l'action vers les inavouables profondeurs de la vie abyssale, annoncent déjà Héloïse, la femme fatale, le vampire qui hante le métro parisien. On notera que Héloïse porte dans son corps la lettre «S», cette lettre qui est la même vue de l'autre côté du miroir. $\mathrm{Ce}$ «S» fatidique («lettre de la déviance» ainsi que l'a désignée Barthes à propos de Sarrazine) évoque ici à la fois le serpent chtonien et le réseau sinueux des voies de métro, fleuve des enfers que Héloïse sillonne en compagnie du sinistre Bottereau-Belzébuth faisant figure de passeur du Styx.

C'est la culpabilité de la chair, la transgression de l'interdit sexuel qui rend le monde des héros fantomatique et leur fait vivre une aventure qui n'est pas la réalité familière et observable. Personne n'a encore analysé l'oeuvre hébertienne sous l'angle du fantastique et pourtant avec Kamouraska, les Enfants du Sabbat et surtout Héloïse, on peut dire qu'Anne Hébert a résolument franchi les frontières du fantastique, genre qui répond étrangement aux mythes personnels de l'auteure et lui impose ses thèmes et ses situations dramatiques: l'intervention du monde du rêve dans la réalité quotidienne, les problèmes de l'enfermement (tombeau - caveau - cimetière - cellule - «chambre de bois»), le pacte avec le diable, la mort personnifiée, les fantômes, les vampires, le monde des vivants et celui des morts où les héros continuent à vivre une vie pâlie et effacée qui ressemble au sommeil.

Dans ce monde de la dualité, la chute aux enfers implique automatiquement une «montée» vers le ciel dans une vaine tentative de purification. «Chute» et «montée», explique Maurice Émond, sont en effet les deux aspects de la punition lourde du poids de la faute, et les gestes des héros, tendus vers la lumière inaccessible, trahissent alors «le désir de verticalité». Le rêve et la fenêtre, «regard supplémentaire et nouvelle vision», deviennent un moyen d'évasion hors de l'univers cloisonné, tout comme, à un moment donné, ils peuvent symboliser à nouveau les cauchemars de la nuit et la séquestration. Car le blanc ne peut jamais totalement triompher du noir, ni de la lumière des ténèbres, puisque la femme est «noire» comme le diable. Elle est à l'origine du mal et c'est toujours par elle que la faute est commise. Pour «la femme à la fenêtre» qui rêve et qui s'ennuie, tous les moyens seront bons pour assouvir ses passions et se libérer des liens qui la retiennent prisonnière. Elle est «une bête sauvage», diabolique, qui sème la malédiction partout où elle passe, comme Lia, Élisabeth, Héloïse, mais aussi ne l'oublions pas, comme Nora des Fous de Bassan par qui le péché entre dans 
la communauté, double de cette même Nora du Temps sauvage qui guette les bûcherons, à la sortie du bois, «pour les saigner à blanc comme une belette».

Pour mieux accomplir leur funeste mission, le Diable a doté toutes ces femmes d'une beauté fatale, d'un pouvoir de fascination irrésistible, et qu'elles le veuillent ou non, elles ont signé avec lui un pacte et doivent appliquer «la loi». C'est sur les pas de cette «femme immortelle», de cette «Mère terrible» que Maurice Émond nous conduit d'une main sûre à travers la forêt des signes et des symboles de l'univers imaginaire hébertien. Elle est, écrit-il «la figure maternelle qui incarne les valeurs aliénantes des Claudine, Agnès, Aline, madame Tassy ou Felicity Jones et qui naît au grand jour avec Philomène des Enfants du Sabbat». Jung a expliqué, rappelons-le, cette conception tenace du satanisme de la femme par une réminiscence maintenue vivace dans l'inconscient collectif, aux temps lointains où l'homme vivait dans la peur des forces primitives de la nature et des caprices de sa propre bestialité. Les structures mentales archaïques étaient alors de type matriarcal et démoniaque, d'où ce lien entre la femme et le démon, d'où l'archétype de la «mère terrible».

Il ressort de cet essai que tous les héros hébertiens vivent un drame joué d'avance, tout comme le destin de la communauté de Griffin Creek pouvait se lire dans les lettres tracées sur le mur du presbytère par les jumelles Pat et Pam. C'est pourquoi tout est vécu et senti à l'intérieur de la terreur subjective qui «déforme» la réalité à la manière des «miroirs déformants». À l'instar du jeune François du Torrent, les héros semblent dans l'attente de quelque «tourmente qui balaierait tout, (les) entraînant avec (leur) mère à jamais lié(s) à (leur) destin funeste» (p. 35). C'est en vain qu'ils fourbissent leurs armes pour trancher ces «liens» qui les «ligotent» à leur implaccable destin. Nous croyons que ce n'est pas un hasard si le nom de toutes les héroïnes d'Anne Hébert contiennent les lettres «l», «i», et «e», ou encore «l», «i» et «a», comme Lia et Délia, comme Élisabeth, Julie, Adelaïde, Aline, Aurélie, Hélö̈se, Claudine, Lucie, Florida, Marilda, Felicity et Philomène (dont la première syllabe est déjà si évocatrice). Certes, plus d'un trait commun «lient» toutes ces femmes entre elles. On pourrait même parler de «lien familial». N'ont-elles pas en effet été enfantées par la même "Mère terrible»? La seule qui saura pour ainsi dire «casser le fil» et se libérer, c'est Catherine dont le nom ne comporte pas le «l» fatidique qui «lie» ou qui étrangle.

$\mathrm{Si}$, comme Maurice Émond l'écrit en conclusion, l'univers symbolique d'Anne Hébert n'a pas fini de nous livrer ses secrets, son étude aura au moins prouvé que les images, les symboles, les mythes ou leurs plus complexes dessins deviennent des grilles révélatrices lorsqu'on les applique sur les énigmes des passions, de la vie et de la mort des héros.

1. La Femme à la fenêtre, l'Univers symbolique d'Anne Hébert, dans «les Chambres de bois», Kamouraska et les Enfants du Sabbat, les Presses de l'Université Laval, coll. Vie des lettres québécoises, Québec, 1984, 391 pages. 